\title{
Application of Computer and Technology in EFL Syllabus Design
}

\author{
Isar Gholaminia Tabari \\ English Language Department, Allameh Mohaddes Noori, Institute of Higher Education, Noor, Iran \\ Azadeh Gholaminia Tabari \\ English Language Department, Allameh Mohaddes Noori, Institute of Higher Education, Noor, Iran
}

\begin{abstract}
Since computers were first introduced into educational facilities, foreign language educators have been faced with the problem of integrating high-tech multimedia techniques into a traditional text-based curriculum. As studies of language teaching have pointed out, 'Language teaching tends in practice to be eclectic.... There are not only exceptionally many paths and educational means for arriving at a given educational goal, but there are also very many types of educational materials which can be used to achieve that goal'. For language educators who are trying to incorporate technology into their curricula, the choices seem endless. Yet the quantity, as well as the limitations, of available computer programs does not guarantee that these programs can be successfully integrated into a curriculum.
\end{abstract}

Index Terms - computer, technology, curriculum development

\section{INTRODUCTION}

In common practice, the opposite tends to be true. Many existing language programs only cover specific areas of language learning, such as reading or grammar, without addressing the full scope of language learning skills. Moreover, every program on the market makes certain assumptions regarding the skill level of its users, the best teaching style, and the elements of language that are most important to learn. Though these programs all stress necessary aspects of language learning, trying to arrange them into a meaningful, comprehensive curriculum often creates confusion and redundancy. Because of the lack of compatibility between individual language programs, it is often difficult to incorporate such programs into a complete language-learning experience. The more serious issue of technical incompatibility is no less severe than content problems. Too often, CALL programs designed for one computer platform may not be compatible with others, rendering the program useless for potential users who lack the correct hardware. For example, many CALL materials that are designed for Macintosh computers are not compatible with Windows-based PC's, and vice versa. In an article from the early 1990s which is still pertinent today, Pusack indicates that many

of these compatibility issues arise when software developers try to take advantage of the full capabilities of the platform they happen to be using-incorporating graphics, sound, and video-yet neglect to make the program compatible with other hardware platforms (Pusack 1991).

\section{LITERATURE REVIEW}

Advances in technology may also cause programs to become incompatible with the computer for which they were intended. Levy stated that the production of computer-assisted multimedia materials had several recurring problems. Among the problems noted in the case of educational software applications, one of the most pressing was that programs and modules are generally not reused within applications and that there was a lack of shared teaching knowledge between applications. A serious problem exists in the current methodology of developing educational software. Each application is developed independently, and teaching knowledge is hard-coded into individual applications. There is little re-use of teaching code or teaching knowledge between applications because we lack a standard language for representing the knowledge, a standard interface to allow applications to access the knowledge, and a set of tools to allow designers to manipulate the knowledge. Due to a lack of technical support, learning how to develop multimedia materials can be a frustrating and even discouraging task. Although technology has been widely adopted and supported in educational systems for over a decade, there are many schools that lack the resources to support CALL development. Beyond these initial difficulties, many larger issues compound the problems associated with independent multimedia development. Because there is so little shared knowledge in the field of courseware development, faculty who produce multimedia materials usually start the development process from scratch, building all necessary coding, scripting, and digitizing and editing audio-visual materials on their own. Yet many institutions are unwilling to support such endeavors, especially on an individual level. As Stephen Ehrmann (2003) notes, courseware development requires just as much investment by the school system as it does by the individual teacher. Changing a course, especially to integrate technology, involves shifts to unfamiliar materials, the creation of new types of assignments, and the invention of new 
ways of assessing student learning. Such a shift poses a great risk to an institution. The steep learning curve, time requirements, and availability of funding dictate the development of courseware, and as a result, projects almost always remain small scale and are rarely developed into full curricula. In addition, the quickening pace of technology makes software development a risky game; it takes so long to develop a comprehensive multimedia program that software often becomes obsolete before it has been fully integrated into curricula. To further exacerbate the problem, small-scale projects are often only suitable to the needs of the faculty developer and are difficult or impossible to adapt even by other faculty members at the same institution. Because expected foreign language skill levels differ from student to student, class to class, and university to university, development of a one-size-fits-all courseware is a misguided and impossible project. Adaptability is key. Without it, programs are unsuitable for extended use. Because of their limitations, most computer language-learning materials are never seen beyond the confines of the campus where they were created.

Writing about the need for teacher training in technology, Teacher laments the fact that 'the mere availability of technologies like the Internet does not automatically translate into enhanced learning experiences, particularly when student and teacher training are lacking' According to Tognozzi, inadequate training, the fear of computers, the lack of technical knowledge, as well as issues of teaching loads and intellectual property rights are among the reasons which prevent teachers from using computers as language-learning tools (2001:487; cf. Lam: 2000:395). Furthermore, she adds, the fact that technology used in foreign language training is constantly changing simply heightens 'confusion and fear among educators' (2001: 487). Those of us who teach language, particularly those involved in the training of future teachers, are acutely aware of the problems posed by the situation Tognozzi describes. There is an urgent need to train teachers and future teachers1 to incorporate new technologies into the language curriculum. Researchers in pedagogy for the teaching of Italian have regularly discussed the need to train those who teach Italian as a foreign language how to evaluate instructional software for effective use in the classroom. Training teacher candidates in CALL is an essential first step in bridging theory and practice. Not only will appropriate training allow teachers to overcome any fear of computers born of a lack of technical knowledge, it will also give them the ability to adapt to the constantly changing technologies for language teaching. The reward potential of technology hinges on its efficacious use, which can take place only if both the instructor and the student have control over the medium. The training itself should incorporate two fundamental stages: 1) the preparation of teachers in effectively evaluating language technologies (software, courseware, and language websites); and 2) the training of teachers to become users of such technologies and to be actively involved in their creation or modification. Training teachers to evaluate language technologies effectively is fundamental in an ever increasing student-centered teaching approach to foreign-language learning. In this environment the role of teachers has changed; their new role is that of guides, coaches, interlocutors, mediators, evaluators, resource advisors, and facilitators. New technologies are compelling teachers to guide the learning path of their students through activities which are gradually more difficult and complex. Even though this new role has been commonly accepted in the foreign language teaching profession for over a decade, teaching is often reduced to lecturing, to a simple communication of information, to a teacher-fronted lesson. Such an approach is definitely not the most productive for students. Cremascoli (1998) has made the point that one of the problems in pedagogy today lies in the very formation of teachers and in the fact that they are too often attached to a historically determined model of transmission of knowledge and fundamentally tied to the practice of a face-to-face or teacher-fronted lesson. Our role as teachers in the technological age is not only to impart new knowledge, but to give students the tools to acquire knowledge, to recognize the value of what they see in books and software as well as on the Internet. As Murphy points out: 'The emphasis in the new era of language learning is on construction as opposed to transmission of knowledge.' A teacher cannot be simply a 'knowledge dispenser.' He or she must be a 'facilitator' as well. In today's view, the teacher is transformed into a coach and consultant on the strategies used to solve problems. Teachers will not be able to fulfill their new role and guide students if they are uncomfortable with new technologies and unable to evaluate them properly. As CALL pedagogues it is our duty to focus the critical abilities of our students on the uses of technology at every level of study and training. Teachers and prospective teachers need a greater appreciation of the communicative and interactive elements of teaching. Learners/teachers who are able to apply the theories they study to the creation of technological aids for instruction will take a more active part in the learning/teaching process, which consequently should increase their motivation in using technology in their own classroom. We need to apply the same principles when training teachers; we need to motivate instructors at every point in their training by showing them how technology can make them more successful instructors.

\section{ARgumentation}

Teachers need also to understand that there is little difference between evaluating technological resources and evaluating traditional resources given that the pedagogical principles are the same. The second phase of training consists of encouraging teachers to become actively involved in the creation or modification of new courseware/software. My own students report that experimenting with software requires a great deal of concentration and practice. However, they also note that any drawbacks are counterbalanced by:

A. the satisfaction of creating authentic and pedagogically useful lessons with the Technology;

B. the ability to deliver lessons which are both pedagogically sound and interesting; 
C. the opportunity to be innovative and creative in planning lessons.

We need to appeal to these motivational elements to encourage teachers to create their own materials. However, active involvement does not necessarily mean that teachers must become programmers or graphic artists. What they do need is a clear understanding of the way different technologies work so that they can give pedagogically sound input to the creators of the new courseware. In an interview with teachers emphasize the need for language teachers to be more actively involved in creating the technological resources they will use in the classroom. The task of creating pedagogically sound and effective courseware cannot be left to programmers with little or no expertise in secondlanguage education. Nor can the task be left to our teaching assistants or part-time language instructors who, even though fluent in the language, do not necessarily have a sound foundation in language pedagogy. If course software is going to be effective it must function in pedagogically appropriate ways. If that is to happen, then trained pedagogues must be involved in establishing evaluative criteria for software (Bancheri: 1997:499-502). In the past few years, the situation has improved somewhat, but not significantly. Didactics is still a "servant of technology," and pedagogy often plays second fiddle to programming. Many of the so-called second-language software, courseware or websites are lacking basic language-learning principles because the procedure to create L2 technologies is often initiated not by the users (teachers) but by software companies, which are largely interested in making a profit (Bancheri: 1997:501). As Benyonet al. point out: 'We have also found that current tools are severely lacking in a number of important respectsparticularly with respect to the design of pedagogically sound courseware' (1999:197). In spite of the advantages of multimedia for language learning, Warschauer argues that there are problems related to its use for language teaching. The lack of programs based on sound pedagogical principles combined with the lack of interactivity and intelligence of these programs limit the ability of multimedia technology to allow for the integration of meaningful and authentic communication. Hanson-

Smith (1997) argues in a similar vein about the lack of an "appropriate pedagogy" of multimedia whereby the media aspects often drive the content rather than the other way around.

New technologies for language teaching should be the fruit of the collaboration between an expert programmer, a graphic artist, and a teacher trained in second-language pedagogy (Hendricks: 1998:216). In such collaboration no compromise should exist as far as the essential didactic elements of the program. The system I use to solve this problem comprises two steps. The first step consists of the identification of some possible student errors so that every time a student supplies a wrong answer which has been anticipated, he or she will receive recorded feedback specifically tailored to that mistake. The second step consists of recording all of the students' wrong answers in what I call the "bank of errors." These answers can be added with an individualized comment to the original database. The process, especially in its second step, is continuous and never ending, but after a while the database will become very solid and direct in its ability to supply individualized feedback. Furthermore, the bank of errors can also be used to create new activities, thus giving the teacher the opportunity to tailor the courseware to target specific areas of weakness. The creation of CALL materials should be an extension of the methods, techniques, and theories used in the language classroom, adapting pedagogically effective techniques to a new medium. The first step in the process is to envisage the same exercise or activity in a classroom without a computer, analyze it in all its different aspects and steps, and then imitate the same procedure in a computerized setting. Furthermore, in creating any computerized activity, I consider not only the teacher's perspective, i.e., the pedagogical view, but also the different perspectives of the student, the software developer, the programmer, and the graphic artist. Viewing courseware development from the teacher's perspective insures that all the pedagogical elements of the activity (in its computerized and non-computerized. form) are analyzed carefully to ensure its pedagogical soundness. To illustrate, I will reconstruct the pedagogical choices and structure I adopted for Testmaker, a computerized program which allows language instructors (Italian, Spanish, French, Chinese, Arabic, etc.) to create and administer quizzes, tests, final exams, etc., and which I use for in-class tests in my language courses. The advantage of creating a computerized testing program for language courses stems first from the need to maximize the use of the teacher's time and resources and, second, to give students an opportunity to better prepare for their exams. The decision to use a non-web-based application is tied to pedagogical issues. Web-based testing applications are generally less secure, and therefore there is a stronger possibility of students' falsifying the results. Moreover response time on the Web is somewhat slower, with a consequent possible loss of student concentration and motivation. Finally, the implementation of what I call the "circular" structure of the test-which I consider a pedagogical priority in this activity - is more difficult to apply in a web-based application. The circular structure works as follows: In Test maker, as in a regular test, the student can start with the last exercise, answer a few questions, then go to the second exercise, answer other questions, then move to the first exercise, and so on. The computer will remember each answer the student has given. This format lowers students' anxiety because they have more time to consider each answer carefully without feeling pressed for time, and they can revisit at any time the questions that they find more challenging or on which they had previously drawn a blank. It illustrates the circular structure of Test maker. The program also includes several other elements which directly or indirectly promote effective teaching: online help; and a button indicating "Time Left;" which only appears on mouse-over, thereby hopefully decreasing the anxiety a student might feel if faced with a ticking clock. Motivation is an important element in language learning which can be fostered in software, courseware and language web pages through appropriate feedback, transparency, and variety. Feedback 
In commercially available software/courseware, the first element to be compromised is often the "feedback," a fundamental element in language teaching. Although creating personalized and precise feedback in grammar exercises, for example, can be very time consuming for programmers (and also for teachers), effective feedback is nonetheless an essential part of the learning process. Our students improve their language skills through specific and individualized feedback (Bancheri: 1997). "Transparency" is more than user-friendliness. Rather, a program should be considered "transparent" when there is "navigation by intuition," where a student-user can use it automatically. These buttons eliminate commands for accented vowels which often constitute an obstacle to concentration and increase a student's anxiety level and they ignore the mechanics of the program and fully concentrate on the language task. As Hendricks cautions: the 'integration of multimedia should be seamless,' as a clumsy and tedious navigational interface will distract from the learning activity and 'its inherent power as a learning tool is handicapped' (1998). In Test maker, for instance, there are buttons which a student can use to type accented vowels automatically. Selecting a variety of activity types not only boosts student interest and motivation it supports a variety of learning styles as well. An obvious way to provide variety is by using audio, video, and graphic sources, which could also be used for a visual or auditory presentation of culture. One needs to find, however, a balance between the immediacy of the information and its multi-mediality. Especially in web-based programs these elements may slow the program down with the consequent risk of distracting the students. All of the activities in Test maker are audio, graphic, video and text-based; they all offer immediate and direct feedback; they are based on specific semantic fields and presented within a cultural context. For this reason, I have created computerized activities using: both linear and circular structures; clickable cultural pages; clickable text; audio dialogues; clickable audio, video, and graphics; dictionaries for semantic fields; crossword puzzles; word searches; word- and sentence-level hangman, word- and sentence-based scramble modules; psychological tests; knowledge tests; personality tests; interactive dictation; dialogue reconstructions; text-, audio-, graphic, and videomatching games; video puzzles; paragraph builders; compositions; open-question modules; and the typical multiplechoice and fill-in-the-blank templates. Each of the activities mentioned has been carefully considered from a pedagogical perspective and has been incorporated, whenever possible, within a cultural context and a specific semantic field. Let me briefly comment on these pedagogical implementations. In the clickable culture pages, students are presented with cultural notes on a specific topic; when they click on a selected highlighted word, the program will respond with a grammatical, vocabulary or cultural explanation with either text, graphics, audio or video. The clickable text and audio dialogues offer the same features. The feedback fosters student reading and listening skills, promotes cultural understanding, and appeals to different learning styles. The goal of clickable audio/video/graphics semantic fields, dictionaries, crosswords and word searches, word- and sentence-hangman/scramble modules is to build and test students' lexical skills. In the clickable dictionaries, students are presented with words, expressions, or full sentences on a specific semantic field. They will be offered a translation, with grammatical or cultural explanations, if appropriate; pronunciation through an audio file; images illustrating the object or the action, whenever appropriate; a video dialogue in which the word, expression or sentence is used in a contextualized situation. The definitions in the crosswords are also presented with either text, audio, graphics and video. The hangman, word searches and scramble modules deal not only with words, but also with expressions and full sentences drawn from the specific semantic field. The psychological/personality tests as well as the tests of knowledge are used often as warm-up activities and give the instructor the opportunity to introduce vocabulary, culture, cultural differences in the specific semantic field discussed. Even though the program which runs the actual test in Test maker is fairly complex and takes advantage of the multimedia features of instructional technologies, the creation process is transparent in that the instructor has to prepare only two text-files. The first contains the test questions, score, answers, etc; the other controls the parameters for the test, such as the time allocated for the completion of the test, the path to save the results, the order in which the questions are presented, the manner in which the answers and score results are presented to the student, etc. All that is required to create a computerized activity from a text-based file is a simple click of the mouse. The ease of creating tests and activities should encourage even the most computer-phobic instructor to supplement classroom instruction with CALL materials. Some of the questions one should ask in applying this perspective are: how are the students going to react to this activity? Is this computerized activity a mere duplication of another activity that could be done with traditional methods in a regular classroom setting? If so, how would the computerized form of the activity be more beneficial to the students? How would the students carry out this activity in a regular classroom setting, and how could I imitate it in a computerized environment? To apply a students' perspective, one needs not only to keep open all channels of communication with them before, during, and after the activities, but also to translate their feedback into programming features.

Students need to concentrate on the activity, not on how the computer works. Transparency will lower the student's affective filters. The student should be familiar with the format of the activity. For example, in testing activities, I prefer a circular format instead of a linear one because, as in regular pen and-paper tests, it will allow test-takers to start with any task and review, compare, modify, or completely change answers at any time and as many times as they like during the test. Students need to feel at ease with the process and content of the activities, especially when they will be assigned "official" marks. An assessment program might comprise the actual tests as well as offer students the possibility of preparing for a test using the same database of activities in different modes. For example, a study-mode can be offered in addition to a test-mode. The study-mode gives students individualized and immediate feedback on 
their answers, their score, a report with all their answers, which can be printed or saved, and the possibility to check the right answers after the completion of the activity. Such a system could offer students the possibility of creating their own study/test-mode activities and therefore to work only on those sections where they need more practice. These features aim once again at lowering student anxiety by creating a familiar environment, and by allowing them to work at their own pace with actual testing items. One of the possible features in the template applications envisioned here is the collection of the data produced by the students. A student's answers can be recorded, with a score, if applicable, along with the time needed to complete each activity.

When students use the composition module, as previously discussed, their output is saved and analyzed; among the data collected is a list of all the different words used and a list of words which have been possibly misspelled. All the files are saved in a secure place on the server, which the instructor can access instantly from his or her own computer. The collected data may be used to improve and update existing materials or to create new activities according to the students' needs (or errors) as evident from the stored files. In grammar tasks, for example, students' wrong responses already saved in the database may be edited so that the students will get a personalized feedback whenever they make the same error. Students' answers can also be used to correct typographical mistakes in the database or to supply an additional answer which was not included in the original database. Obviously, this process requires a lot of dedication and can be very time-consuming, but it can also provide valuable information for both the teacher and the researcher (for a discussion of the usefulness of logs in student learning, In creating applications for language teaching and learning, it is important that the teacher and the programmer establish a strong working relationship. The programmer needs to understand the pedagogical issues, and the teacher needs to be able to envisage the proposed computerized activity from a programmer's perspective. Designing and creating a multimedia CALL package is an extremely demanding task, calling upon a range of skills and meticulous attention to detail. Such is the complexity of computer programs these days that it is highly unlikely that a single person will have all the necessary skills to undertake a CALL development project alone. Team work is therefore essential, and each member of the team must have some understanding of the roles of the other members of the team ... The language teacher who joins a software development team, for example, does not have to possess computer programming skills but he/she must have some understanding of basic programming concepts. Similarly, those responsible for the programming do not have to have knowledge of foreign languages, but they need a good understanding of natural language processing.

Today, people of all ages and backgrounds are seeking to enhance their language experience with multimedia tools. There is an enormous demand for new technology that can blend with traditional teaching methods in order to create an exciting, versatile language-learning environment. The burden is on educators to develop effective courseware that is both pedagogically sound and technologically sophisticated.

\section{CONCLUSION}

Despite the significant demand there is still a marked shortage of high quality, curriculum-based multimedia courseware available to foreign language educators. Moreover, there has been much criticism of the Computer-Assisted Language Learning (CALL) materials that have been produced. Most programs are limited in scope and structure, making them less than ideal as supplements to language curricula. These programs are plagued by a number of problems, in particular a general lack of program reusability, adaptability, and compatibility. For all but the most technically proficient language instructor, CALL programs that cannot be readily integrated into the syllabi of language courses are of very little use.

\section{REFERENCES}

[1] Anderson, D.K., \& Reed, M.W. (1998). The effects of internet instruction, prior computer experience and learning styles on teachers' internet attitudes and knowledge. Journal of Educational Computing Research, 19(3), 227-246.

[2] Benyon, D., Stone, D., \&Woodroffem M. (1997). Experience with developing multimedia courseware for the World Wide Web: The need for better tools and clear pedagogy. Retrieved on April, $25^{\text {th }}$ [Online]. Available: http://ijhcs.open.ac.uk/benyon/benyon.pdf.

[3] Chickering, A., \&Ehrmann, S. (2002). Implementing the seven principles: Technology as lever. Retrieved on April, 25 ${ }^{\text {th }}$ [Online]. Available: http://www.tltgroup.org/programs/seven.html.

[4] Czikszentmihalyi, M. (2000). Abstracted by D. Dickinson from Creating the future: Perspectives on educational change (1996), New Horizons for Learning [Online]. Retrieved on April, 25 ${ }^{\text {th }}$ Available: http://www.newhorizons.org/future/Creating_the_Future/crfut_csikszent.html.

[5] Murphy, E. (2000). Strangers in a strange land: Teachers' beliefs about teaching and learning French as a second or foreign language in online learning environments [Online]. Retrieved on April, 25. Available: http://www.ucs.mun.ca/ emurphy/strangers/toc.html.

[6] Tsui, A.B.M., \&Fullilove, J. (1998). Bottom-up or top-down processing as a discriminator of L2 listening performance. Applied Linguistics, 19(4), 432-451.

[7] Warschauer, M. (Ed.) (1995).Virtual connections: On-line activities and projects for networking language learners. Honolulu: Second Language Teaching and Curriculum Center, U of Hawai'i.

[8] Warschauer, M. (1996). Computer-assisted language learning: An introduction. In S. Fotos (Ed.), Multimedia Language Teaching (pp. 3-20). Tokyo: Logos International. 


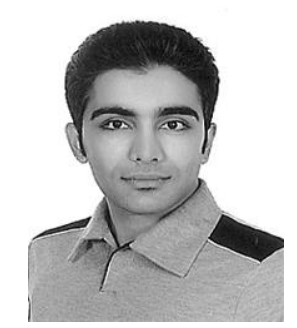

Isar Gholaminia Tabari was born in Babol, Iran in 16/09/1985. He received his B.A degree in the field of English translation from Qaemshahr Mazandaran, Iran in 2007. And he received his M.A degree in Language teaching from Garmsar Azad University in Garmsar, Semnan, Iran, in 2010.

He has been teaching English language at Iranmehr English Language Institute, and now he is a freelance instructor at Sama Institute of higher education, Alameh Mohaddes Noori Institute of higher education and Parsa Institute of higher education in Babolsar, Mazandaran, Iran. His research interests include language learning strategies.

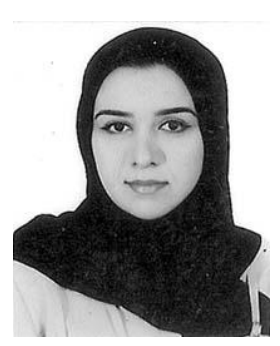

Azadeh Gholaminia Tabari was born in Babol, Iran in 19/09/1982.

She received her B.A degree in the field of English translation from Qaemshahr University in Qaemshahr, Mazandaran, Iran, in 2004. And she received her M.A degree in the field of teaching English from Mazandaran (Pardis) University in Babolsar, Mazandaran, Iran, in 2007.

She has been teaching English Language at Iran-Canada English Language Institute, Sama Institute of higher education and now she is the member of English Language faculty at Allameh Mohaddes Noori Institute of higher education in Noor, Iran. She has published an article by the name of Challenges of Language Syllabus Design in EFL/ESL Contexts in Journal of Language Teaching and Research, Finland, Academy Publisher, 2013. Moreover her research interests include Language teaching strategies. 